\title{
Caracterização molecular dos fatores de virulência de estirpes de Escherichia coli isoladas de papagaios com colibacilose aviária
}

Terezinha KNÖBL ${ }^{1}$

Silvia Nery GODOY²

Eliana Reiko MATUSHIMA²

Marta Brito GUIMARÃES²

Antonio José Piantino

FERREIRA ${ }^{2}$

\section{Correspondência para:}

Terezinha Knöbl - Laboratório de EpidemiologiaeDoenças Infecciosas-FMU. Avenida Santo Amaro 1239, Vila Nova Conceição, São Paulo, SP. Cep 04505-002. E-mail:tknobl@fmu.br

Recebido para publicação: 07/04/2007 Aprovado para publicação: 29/07/2008

\author{
1 - Faculdade de Medicina Veterinária do Centro Universitário das Faculdades \\ Metropolitanas Unidas, São Paulo - SP \\ 2 - Departamento de Patologia da Faculdade de Medicina Veterinária e Zootecnia \\ da Universidade de São Paulo, São Paulo - SP
}

\section{Resumo}

Oito amostras de Escherichia coli isoladas de papagaios com colibacilose aviária foram sorogrupadas e investigadas para a presença dos fatores de virulência: pili associado a pielonefrite (pap), fímbria S (sfa), adesina afimbrial (afa), cápsula K1 (neu), curli (crl, csgA), hemaglutinina termosensível (tsh), enterotoxinas termo-lábil (LT) e termo-estável (STa e STb), Shiga-like toxinas (Stx1 e Stx2), Fator citotóxico necrotizante (cnfl), hemolisina (bly), aerobactina (iuc) e resistência sérica (iss). Os resultados mostraram que os isolados pertenciam a seis sorogrupos: O23; O54; O64; O76; O128 e O152. Os genes de virulência detectados foram: $c r^{+}$em todos os isolados; $p a p^{+} ; i s s^{+}$e $i u c^{+}$em três isolados, $t s h^{+}$ em dois isolados. Todas as amostras foram negativas para os genes neu, csgA, sfa, afa, bly, cnf e para as toxinas LT, STa, STb, Stx1 e Stx2. Estes resultados sugerem que amostras de E. coli isoladas de papagaios apresentam alguns fatores de virulência das amostras do patotipo de E. coli patogênica para aves (APEC).

\section{Introdução}

As infecções bacterianas figuram entre as principais causas de enterite e septicemia em aves silvestres, com destaque para os membros da família Enterobacteriaceae..$^{1}$ Muitos autores consideram a presença de microrganismos Gramnegativos no trato digestório de psitacídeos como um fator indesejável, devido ao potencial de patogenicidade destas bactérias para as aves. ${ }^{1,2,3}$ Escherichia é o gênero mais comumente encontrado da família das Enterobactérias e a espécie Escherichia coli, o agente mais freqüentemente isolado em tecidos obtidos na necrópsia de aves silvestres. ${ }^{4,5,6}$

A colonização intestinal das aves por amostras de E. coli patogênicas está intimamente associada a fatores de estresse, má nutrição ou problemas de manejo ambiental. ${ }^{7}$ As manifestações clínicas da doença incluem sinais de intensa prostração, enterite, hepatite, hepato e esplenomegalia, aerossaculite, poliserosite e comprometimento de diversos órgãos como rins, oviduto, pulmão, medula óssea e articulações. ${ }^{8}$

A severidade do quadro clínico depende do potencial de virulência do agente que é determinado pelo conjunto de genes localizado nas ilhas de patogenicidade. ${ }^{9,10}$ Estudos envolvendo patotipos de E. coli patogênicos para galinhas (APEC) apontam os fenômenos de aderência bacteriana, crescimento em condições de restrição do íon ferro e a resistência sérica como principais responsáveis pela patogenia da doença. ${ }^{11}$

$A$ aderência bacteriana na maioria das vezes é mediada por adesinas fimbriais manose-sensíveis (pili tipo I) e manoseresistentes (fímbrias $\mathrm{P}$ e S). ${ }^{12}$ A capacidade de crescimento em meios com ferro indisponível resulta da produção de substâncias quelantes, denominadas sideróforos. Amostras de E. coli aviárias 
usualmente seqüestram o ferro através da produção de aerobactina. ${ }^{13}$

A habilidade em resistir aos fatores séricos inibitórios permite que a bactéria escape da ação do sistema complemento e da fagocitose nos processos de infecção sistêmica. A resistência sérica das bactérias tem sido atribuída a diversas estruturas destas células incluindo o lipopolissacarídeo (LPS), a cápsula $\mathrm{K} 1$ e as proteínas de membrana externa (OMPs) que são codificadas pelos genes tra $\mathrm{T}$ e $i s{ }^{13,14}$

O objetivo deste trabalho foi caracterizar amostras de E. coli isoladas de papagaios com colibacilose, sob o ponto de vista molecular e de virulência.

\section{Material e Método}

\section{Origem das amostras de Escherichia coli}

Este estudo foi realizado a partir de amostras de E. coli isoladas de oito papagaios doentes, atendidos pelo Ambulatório de Aves do Hospital da Faculdade de Medicina Veterinária da Universidade de São Paulo. As aves envolvidas apresentavam manifestações clínicas compatíveis com o quadro de colibacilose aviária e evoluíram para o óbito.

Fragmentos de fígado e coração foram colhidos com assepsia durante a necropsia, para realização da cultura. As amostras foram transportadas ao Laboratório de Ornitopatologia FMVZUSP, sob refrigeração.

A cultura foi realizada após cultivo em caldo infusão de cérebro e coração (BHI) a $37^{\circ} \mathrm{C}$ por 18 horas e o plaqueamento em ágar sangue e ágar MacConkey, incubando-se a $37^{\circ} \mathrm{C}$ por 24 horas, em aerobiose. ${ }^{15} \mathrm{Em}$ todas as culturas realizadas, obteve-se apenas o crescimento de colônias fermentadoras de lactose, identificadas posteriormente pela série bioquímica (Enterokit -Probac ${ }^{\circledR}$ ) como Escherichia coli. As amostras isoladas foram subcultivadas e estocadas a $-20^{\circ} \mathrm{C}$ em meio BHI com 15\% de glicerol.
Uma cepa de E. coli K12 foi utilizada como controle negativo. As cepas EC27 (sorogrupo O78) e EC20 (sorogrupo O6), pertencentes à coleção de culturas de E. coli do Laboratório de Ornitopatologia da USP, foram utilizadas como controles positivos.

\section{Determinação do sorogrupo}

Os sorogrupos foram identificados de acordo com a metodologia descrita por Guinée et al. ${ }^{16}$ utilizando os anticorpos que identificam o antígeno somático $\mathrm{O}$ (O1 a O185). Estes foram produzidos no Laboratório de Referência em E. coli (LREC) - na Universidade de Santiago de Compostela, Lugo, España - http:// www.lugo.usc.es/ecoli.

\section{Caracterização molecular pela Reação em cadeia pela polimerase - PCR}

Diferentes pares de oligonucleotídeos foram utilizados para detectar a presença dos genes que codificam os seguintes fatores de virulência: pili associado à pielonefrite ( $p a p$ ), a-hemolisina (bly), aerobactina (iuc), fator citotóxico necrotizante 1 (cnf 1 ), fimbria S (sfa), adesina afimbrial I (afaI), enterotoxinas termo lábil (LT) e termo estável (STa e $\mathrm{STb}$ ), Shiga-like toxinas (Stx1 e Stx2), adesina regulada pela temperatura $(t s h)$ e curli (crl, $\operatorname{csg} \mathrm{A})$. A tabela 1 ilustra a seqüência de bases dos primers e o tamanho dos fragmentos amplificados esperados. $17,18,19,20,21,22,23,24,25$

A extração de DNA foi realizada de acordo com a metodologia descrita por Boom et al. ${ }^{26}$. A solução para amplificação foi composta por Tris- $\mathrm{HCl}$ (pH 8.3) $10 \mathrm{mM}, \mathrm{KCl} 50 \mathrm{mM}, \mathrm{MgCl}_{2} 1,5$ $\mathrm{mM}$, gelatina $0,001 \%$ (água/vol), desoxinucleotídeos trifosfatos $200 \mathrm{mM}$, pares de oligonucleotídeos e enzima Taq DNA polimerase 0,5 $\mathrm{U}$, num volume final de $25 \mathrm{ml}$. Os fragmentos amplificados foram separados por eletroforese em gel de agarose a 1,5\% e corados com Brometo de Etídeo. ${ }^{27}$ 
Tabela 1 - Seqüência de oligonucleotídeos, tamanho dos fragmentos, e referências utilizadas para a deteç̧ão dos fatores de virulência de E. coli. São Paulo, 2007

\begin{tabular}{|c|c|c|c|}
\hline $\begin{array}{c}\text { Fator de virulência } \\
\text { (Gene) }\end{array}$ & Seqüência dos oligonucleotídeos $\left(5^{\prime}-3^{\prime}\right)$ & $\begin{array}{l}\text { Fragmento } \\
\text { amplificado } \\
\text { (pb) }\end{array}$ & Referências \\
\hline $\begin{array}{c}\text { Adesina } \mathrm{P} \\
\text { (pap) }\end{array}$ & $\begin{array}{l}\text { GCAACAGCAACGCTG GTTGCATCAT } \\
\text { AGAGAGAGCCACTCTTATACGGACA }\end{array}$ & 336 & Yamamoto et al. ${ }^{17}$ \\
\hline $\begin{array}{c}\text { Adesina S } \\
\text { (sfa) }\end{array}$ & $\begin{array}{l}\text { CTCCGGAGAACTGGGTGCATCTTAC } \\
\text { CGGAGGAGTAATTACAAACCTGGCA }\end{array}$ & 410 & Yamamoto et al. ${ }^{17}$ \\
\hline $\begin{array}{l}\text { Adesina afimbrial } \\
\qquad(a f a)\end{array}$ & $\begin{array}{l}\text { GCTGGGCAGCAAACTGATAACCTC } \\
\text { CATCAAGCTGTTTGTTCGTCCGCCG }\end{array}$ & 750 & Yamamoto et al. ${ }^{17}$ \\
\hline $\begin{array}{l}\text { Hemolisina } \\
\text { (hly) }\end{array}$ & $\begin{array}{l}\text { AACAAGGATAAGCACTGTTCTGGCT } \\
\text { ACCATATAAGCGGTCATTCCCGTCA }\end{array}$ & 1177 & Yamamoto et al. ${ }^{17}$ \\
\hline $\begin{array}{l}\text { Aerobactina } \\
\text { (iuc) }\end{array}$ & $\begin{array}{l}\text { TACCGGATTGTCATATGCAGACCGT } \\
\text { AATATCTTCCTCCAGTCCGGAGAAG }\end{array}$ & 602 & Yamamoto et al. ${ }^{17}$ \\
\hline $\begin{array}{l}\text { Fator citotóxico } \\
\text { necrotizante } \\
\text { (cnfl) }\end{array}$ & $\begin{array}{l}\text { AAGATGGAGTTTCCTATGCAGGAG } \\
\text { CATTCAGAGTCCTGCCCTCATTATT }\end{array}$ & 498 & Yamamoto et al. ${ }^{17}$ \\
\hline Enterotoxina LT & $\begin{array}{l}\text { GGCGACAGATTATACCGTGC } \\
\text { CCGAATTCTGTTATATATGTC }\end{array}$ & 696 & Schultsz et al. ${ }^{18}$ \\
\hline Enterotoxina STa & $\begin{array}{l}\text { TTAATAGCACCCGGTACAAGCAGG } \\
\text { CTTGACTCTTCAAAAGAGAAAATTAC }\end{array}$ & 147 & Olsivk et al. ${ }^{19}$ \\
\hline Enterotoxina $\mathrm{STb}$ & $\begin{array}{l}\text { ATCGCATTTCTTCTTGCATC } \\
\text { GGGCGCCAAAGCATGCTCC }\end{array}$ & 172 & Blanco et al. 20 \\
\hline Toxina Stx1 & $\begin{array}{l}\text { GAAGAGTCCGTGGGATTACG } \\
\text { AGCGATGCAGCTATTAATAA }\end{array}$ & 130 & Pollard et al. ${ }^{21}$ \\
\hline Toxina Stx2 & $\begin{array}{l}\text { CCGTCAGGACTGTCTGAAAC } \\
\text { GAGTCTGACAGGCAACTGTC }\end{array}$ & 726 & Woodward et al. ${ }^{22}$ \\
\hline $\begin{array}{l}\text { Curli } \\
(\mathrm{crl})\end{array}$ & $\begin{array}{l}\text { TTTCGATTGTCTGGCTGTATG } \\
\text { CTTCAGATTCAGCGTCGTC }\end{array}$ & 250 & Maurer et al. ${ }^{23}$ \\
\hline $\begin{array}{c}\text { Curli } \\
(\operatorname{csgA})\end{array}$ & $\begin{array}{l}\text { ACTCTGACTTGACTATTACC } \\
\text { AGATGCAGTCTGGTCAAC }\end{array}$ & 200 & Maurer et al. ${ }^{23}$ \\
\hline $\begin{array}{c}\text { Hemaglutinina } \\
\text { termo-sensivel } \\
\text { (tsh) }\end{array}$ & $\begin{array}{l}\text { GGGAAATGACCTGAATGCTGG } \\
\text { CCGCTCATCAGTCAGTACCAC }\end{array}$ & 420 & Maurer et al. ${ }^{23}$ \\
\hline $\begin{array}{l}\text { Cápsula K1 } \\
\text { (neu) }\end{array}$ & $\begin{array}{l}\text { TATAATTAGTAACCTGGGGC } \\
\text { GGCGCTATTGAATAAGACTG }\end{array}$ & 927 & Tsukamoto et al. ${ }^{24}$ \\
\hline $\begin{array}{l}\text { Resistência sérica } \\
\text { (iss) }\end{array}$ & $\begin{array}{l}\text { GTGGCGAAAACTAGTAAAACAGC } \\
\text { CGCCTCGGGGTGGATAA }\end{array}$ & 760 & Horne et al. ${ }^{25}$ \\
\hline
\end{tabular}

\section{Resultados}

Os resultados estão descritos na tabela 2. Foram identificados seis sorogrupos distintos: O23; O54; O64; O76; O128 e O152. Uma amostra não teve o sorogrupo identificado.

A presença do gene que codifica a fímbria P (pap) foi detectada em três das oito amostras estudadas. Todas as amostras foram positivas para o gene $\mathrm{crl}$, mas nenhuma amostra $\mathrm{crl}+$ possui o gene responsável pela síntese da subunidade estrutural $(\operatorname{csg} \mathrm{A})$.

Duas amostras foram identificadas como positivas para as adesinas reguladas pela temperatura $(t s h)$. Os genes responsáveis pela captura de ferro (iuc) e pela resistência sérica (iss) foram identificados em três amostras (3/8).

Nenhuma amostra foi positiva para 
Tabela 2 - Sorogrupos e resultados obtidos na PCR para os fatores de virulência de $E$. coli isoladas de papagaios com colibacilose. São Paulo, 2007

\begin{tabular}{|c|c|c|c|c|c|c|c|c|}
\hline $\begin{array}{l}\text { Isolado } \\
\text { (E. coli) }\end{array}$ & $\begin{array}{c}\text { Material } \\
\text { Clínico }\end{array}$ & sorogrupos & $\mathrm{crl}$ & $\operatorname{csg} \mathrm{A}$ & pap & iss & $i u c$ & tsh \\
\hline PSC 1 & Fígado & ONT & + & - & + & + & - & - \\
\hline PSC 2 & Fígado & $\mathrm{O} 152$ & + & - & - & - & - & - \\
\hline PSC 3 & Coração & O64 & + & - & + & + & - & - \\
\hline PSC 4 & Coração & $\mathrm{O} 23$ & + & - & - & + & + & + \\
\hline PSC 5 & Fígado & $\mathrm{O} 128$ & + & - & - & - & - & - \\
\hline PSC 6 & Fígado & O76 & + & - & - & - & + & - \\
\hline PSC 7 & Fígado & O54 & + & - & + & - & + & + \\
\hline \multirow[t]{2}{*}{ PSC 8} & Coração & 0152 & + & - & - & - & - & - \\
\hline & TOTAL & & $8 / 8$ & $0 / 8$ & $3 / 8$ & $3 / 8$ & $3 / 8$ & $2 / 8$ \\
\hline
\end{tabular}

os genes que codificam toxinas, hemolisina, cápsula $\mathrm{K} 1$ e as adesinas $\mathrm{S}$ e afa.

Das oito amostras analisadas, quatro foram positivas para no máximo dois fatores de virulência pesquisados, enquanto outras quatro apresentaram-se positivas para três ou quatro dos seis fatores pesquisados.

\section{Discussão e Conclusões}

Diversos ensaios fenotípicos e genotípicos têm sido descritos para diferenciar amostras de E. coli patogênicas e não patogênicas. ${ }^{2,14,28,29,30,31} \mathrm{O}$ uso de técnicas moleculares, particularmente da PCR, é o modo mais rápido e sensível para detectar fatores de virulência bacteriana. No entanto, a literatura sobre os fatores de virulência de amostras de E. coli isoladas de psitacídeos é escassa. $^{8,11}$

Os dados deste estudo (Tabela 2) mostraram a presença do gene pap em três da oito amostras estudadas. Estes resultados são semelhantes aos descritos por Janben et al. ${ }^{32}$ que identificaram $30 \%$ de amostras pap+ $\mathrm{em}$ isolados de $E$. coli aves comerciais. A fímbria P é uma adesina manose-resistente freqüentemente observada no patotipo UPEC presente em isolados de infecções do trato urinário (cistite e pielonefrite) em humanos. A expressão da fímbria $\mathrm{P}$ em $E$. coli de aves parece estar relacionada à colonização de órgãos internos (fígado e coração) nos estágios mais avançados da colibacilose. ${ }^{33}$

Outro gene identificado neste trabalho foi o $t s h$. Este gene é responsável pela síntese de uma proteína de aderência termo-sensível e com capacidade hemaglutinante, que possui uma alta homologia com IgA proteases de Neisseria gonorrboeae e Haemophilus influenzae. Esta adesina tem sido apontada como um importante marcador de virulência em APEC. ${ }^{31,34}$ Neste trabalho o gene tsh foi identificado em um quarto das amostras estudadas.

Os genes iuc e iss foram detectados no mesmo número de amostras (3/8). Segundo Jaben et al..$^{32}$ e Ngeleka et al..$^{35}$ a virulência das amostras de E.coli em infecções extra-intestinais depende da combinação de fatores de virulência, com particular destaque para os genótipos iuct tsh+ e iuct tsht, pap + , considerados altamente virulentos. Esta combinação de genes foi detectada em duas amostras deste estudo (PSC4 e PSC54), pertencentes aos sorogrupos O23 e O54, respectivamente.

Os sorogrupos identificados neste estudo diferem dos sorogrupos identificados com maior freqüência (O2 e O78) nos quadros de colibacilose aviária em criações de aves comerciais no Brasil descritos por Menão et al. ${ }^{36}$; e dos sorogrupos identificados 
em isolados enteropatogênicos para psitacídeos por Scremmer et al. ${ }^{29}$ (EPEC O110, O63, O131 e O153).

Estudos epidemiológicos sobre os sorogrupos envolvidos nos casos de colibacilose em aves silvestres, bem como um conhecimento mais profundo sobre os fatores de virulência de amostras de E. coli isoladas de psitacídeos com diarréia e septicemia serão de grande auxílio para a conservação destas espécies, além de auxiliarem no diagnóstico de aves doentes, evitando a exposição humana a amostras patogênicas de UPEC e EPEC.

\section{Agradecimentos}

À FAPESP (processo - 05/57500-9), pelo suporte financeiro.

\section{Molecular characterization of virulence factors in Escherichia coli strains isolated from parrots with colibacilosis}

\section{Abstract}

A total of eight Escherichia. coli isolates from psittacine birds were serogrouping and investigated for the virulence factors: pili associated with pyelonephritis (pap), S fimbriae ( $s f a$ ), afimbrial adhesin (afa), capsule K1 (neu), curli fibers (crl, csgA), temperature-sensitive hemagglutinin $(t s h)$, heat labile (LT) and heat stable (STa and STb) enterotoxins, Shiga-like toxins (Stx1 and Stx2), Cytotoxic necrotizing factor (cnf1), haemolysin ( $h$ hy), aerobactin production (iuc) and serum resistance (iss). The results showed that the isolates belonged to six serogroups: O23; O54; O64; O76; O128 and O152. The found virulence genes were: $c r l^{+}$in all isolates, pap $^{+}, i u c^{+}$and $i s s^{+}$in three isolates; $t s h^{+}$in two isolates. All strains were negative for genes neu, $\operatorname{csg} \mathrm{A}, \mathrm{sfa}, a f a, b l y, c n f$ and LT, STa, STb, Stx1, Stx 2 toxins. Our findings suggested that some $E$. coli isolated from psittacine birds present some virulence factors of avian pathogenic E. coli (APEC) pathotype.
Key words:

Parrots. Escherichia coli. Virulence factors. APEC. Colibacilosis.

\section{Referências}

1 HOEFER, H. L. Diseases of the gastrointestinal tract. In: ALTMAN, R. B.; CLUBB, S. L.; DORESTEIN, G. M.; QUSENBERY, K. (Ed.). Avian medicine and surgery. Philadelphia: Saunders Company, 1997. p. 419-453.

2 STYLES, D. K.; FLAMMER, K. Congo red binding of Escherichia coli isolated from the cloacae of psittacine birds. Avian Diseases, v. 35, p. 46-48, 1991.

3 WILLARD, J. G. Common digestive tract disorders. In: SYMPOSIUM ON AVIAN MEDICINE, 1995. Proceedings... 1995. p. 40-52.

4 DORRESTEIN, G. M.; BUITELAAR, M. N.; VAN DER HAGE, M. H.; ZWART, P. Evaluation of a bacteriological and mycological examination of psittacine birds. Avian Diseases, v. 29, n. 4, p. 951 962, 1985.

5 GERLACH, H. Bacteria. In: RITCHIE, B. W.; HARRISON, G. J.; HARRISON, L. B. Avian medicine: principles and application. Florida: Wingers, 1994. p. 949- 983.

6 GODOY, S. N. Patologia comparada de psitacídeos mantidos em cativeiro no Estado de São Paulo. 2001. 214 f. Dissertação (Mestrado) - Faculdade de Medicina Veterinária e Zootecnia, Universidade de São Paulo, São Paulo, 2001.

7 MATTES, B. R.; CONSIGLIO, S. A. S.; ALMEIDA, B. Z.; GUIDO, M. C.; ORSI, R. B.; SILVA, R. M.; COSTA, A.; FERREIRA, A. J. P.; KNÖBL, T. Influência da biossegurança na colonização intestinal por Escherichia coli em psitacídeos. Arquivos do Instituto Biológico, v. 72, n. 1, p. 13-16, 2005.

8 SAIDENBERG, A. B.; KNÖBL, T. Colibacilose em aves ornamentais e silvestres: revisão. Ciência Veterinária nos Trópicos, v. 8, p. 16-28, 2005.

9 HACKER, J.; BLUM-OEHLER, G.; MÜHLDORFER, I.; TSCH ÄPE, H. Pathogenicity island of virulent bacteria: structure, funtion and impact on microbial evolution. Molecular Microbiology, v. 23, p. 1089-1097, 1997.

10 KARIYAWASAM, S.; JOHNSON, T. J.; DEBROY, C.; NOLAN, L. K. Occurrence of pathogenicity island IAPEC-01 genes among Escherichia coli implicated in avian colibacillosis. Avian Diseases, v. 50, p. 405-410, 2006.

11 DHO-MOULIN, M.; FAIRBROTHER, J. M. Avian 
pathogenic Escherichia coli (APEC). Veterinary Research, v. 30, p. 299-316, 1999.

12 KNÖBL, T.; ROCHA, L. T.; ORSI, R. B.; PAIXÃO, R.; MORENO, A. M.; FERREIRA, A. J. P. Investigação molecular dos fatores de virulência de Escherichia coli isoladas de fezes de psitacídeos mantidos em cativeiro. In: CONGRESSO DA ASSOCIAÇÃO BRASILEIRA VETERINÁRIOS DE ANIMAIS SELVAGENS ABRAVAS, 15., Águas de São Pedro. 2006, Anais... Águas de São Pedro: Associação Brasileira dos Veterinários de Animais Selvagens, 2006.

13 MONROY, M. A.; KNÖBL, T.; BOTTINO, J. A.; FERREIRA, C. S.; FERREIRA, A. J. Virulence characteristics of Escherichia coli isolates obtained from broilers breeders with salpingitis. Comparative Immunology, Microbiology and Infectious Diseases, v. 28, n. 1, p. 1-15, 2005.

14 KNÖBL, T.; BACCARO, M. R.; MORENO, A. M.; VIEIRA, M. A.; FERREIRA, C. S.; FERREIRA, A. J. Virulence properties of Escherichia coli isolated from ostriches with respiratory disease. Veterinary Microbiology, v. 2283, n. 1, p. 71-80, 2001.

15 GROSS, W. B. Colibacilosis. In: HOFSTAD, M. S.; CALNEK, B. W.; HELMBOD, C. F.; REID, W. M.; YODER, H. W. Disease of poultry. 3. ed. Ames, lowa State: University Press, 1991. p. 138-144.

16 GUINÉE, P. A.; JANSEN, W. H.; WADSTRÖM, T.; SELLWOOD, R. Escherichia coli associated with neonatal diarrhea in piglets and calves. Current Topics in Veterinary Animal and Science, v. 13, p. 126-162, 1981.

17 YAMAMOTO, S.; TERAI, A.; YURI, K.; KURAZONO, H.; TAKEDA, Y.; YOSHIDA, O. Detection of urovirulence factors in Escherichia coli by multiplex polymerase chain reaction. FEMS Immunology and Medical Microbiology, v. 12, p. 8590, 1995.

18 SCHULTSZ, C.; POOL, G. J.; VAN KETEL, R.; WEVEK, D.; SPEELMAN, P.; DANKERT, J. Detection of enterotoxigenic Escherichia coli in stool samples by using nonradioactively labeled oligonucleotide DNA probes and PCR. Journal of Clinical Microbiology, v. 32, p. 2393-2397, 1994.

19 OLSIVIK, O.; STROCKBINE, N. A. In: PERSING, D. H.; SMITH, T. F.; TENOVER, F.C.; WHITE, T. J. Diagnostic molecular microbiology: principles and applications. Washington: American Society for Microbiology, 1993. p. 271-276.

20 BLANCO, M.; BLANCO, J. E.; GONZALEZ, E. A.; MORA, A.; JANSEN, W.; GOMES, T. A. T.; ZERBINI, F.; YANO, T.; PESTANA DE CASTRO, A. F.; BLANCO, $G$. Genes coding for enterotoxins and verotoxins in porcine Escherichia coli strains belonging to different $\mathrm{O}: \mathrm{K}: \mathrm{H}$ serotypes: relationship with toxic phenotypes. Journal of Clinical Microbiology, v. 35, p. 2958-2963, 1997.

21 POLLARD, D. R.; JOHNSON, W. M.; LIOR, H.;
TYLER, S. D.; ROZES, K. R. Rapid and specific detection of verotoxin genes in Escherichia coli by the polymerase chain reaction. Journal of Clinical Microbiology, v. 28, p. 540-545, 1990.

22 WOODWARD, M. J.; CARROLL, P. J.; WRAY, C. Detection of entero and verocyto-toxin genes in Escherichia coli from diarrhea disease in animals using polymerase chain reaction. Veterinary Microbiology, v. 31, p. 251-261, 1992.

23 MAURER, J. J.; BROWN, T. P.; STEFFENS, W. L.; THAYER, S. G. The occurrence of ambient temperatureregulated adhesins, curli, and the temperature-sensitive hemagglutinin Tsh among avian Escherichia coli. Avian Diseases, v. 42, p. 106-118, 1998.

24 TSUKAMOTO, T. PCR method for detection of K1 antigen and serotypes of Escherichia coli isolated from extraintestinal infection. Kansenshogaku Zashi, v. 71, n. 2, p. 125-129, 1997.

25 HORNE, S. M.; PFAFF-MCDONOUGH, S. J.; GIDDINGS, C. W.; NOLAN, L. K. Cloning and sequencing of the iss gene from a virulent avian Escherichia coli. Avian Diseases, v. 44, p. 179-184, 2000.

26 BOOM, R.; SOL, C. J. A.; SALIMANS, M. M. M.; JANSEN, C. L.; WERTHEIM-VAN DILLEN, P. M. E.; VAN DER NOORDAA, J. Rapid and Simple Method for purification of Nucleic Acids. Journal of Clinical Microbiology, v. 28, p. 495-503, 1990.

27 MANIATIS, T.; FRITSCH, E. F.; SAMBROOK, J. Molecular cloning: a laboratory manual. New York: Cold Spring Harbor, 1982.

28 PAKPINYO, S.; LEY, D. H.; BARNES, J. P.; VAILLANCOURT, J. P.; GUY, J. S. Prevalence of enteropathogenic Escherichia coli in naturally occurring cases of poult enteritis-mortality syndrome. Avian Diseases, v. 46, p. 360-369, 2002.

29 SCHREMMER, C.; LOHR, J. E.; WASTLHUBER, U.; KÖSTERS, J.; RAVELSHOFER, K.; STEINRÜCK, H.; WIELER, L. H. Enteropathogenic Escherichia coli in psittaciformes. Avian Pathology, v. 28, p. 349-354, 1999.

30 WASTLHUBER, U.; SPLEISS, C.; LOHR, J. E. Verotoxin production and adhesion genes of $E$. coli isolates from commercial poultry and psittacines: detection by PCR. Tierarztl Prax Ausg G Grosstiere Nutztiere, v. 26, n. 1, p. 49-52, 1998.

31 STATHOPOULUS, C.; PROVENCE, D. L.; CURTISS, R. Characterization of the avian pathogenic Escherichia coli hemagglutinin Tsh, a member of the immunoglobulin A protease-type family of autotransporters. Infection and Immunity, v. 67, p. 772-781, 1999.

32 JANBEN, T.; SCHWARZ, C.; PREIKSCHAT, P.; VOSS, M.; PHILIPP, H. C.; WIELER, L. H. Virulenceassociated genes in avian pathogenic Escherichia coli (APEC) isolated from internal organs of poultry having 
died from colibacillosis. International Journal of Medical Microbiology, v. 291, p. 371-378, 2001.

33 POURBAKHSH, S. A.; DHO-MOULIN, M.; BRÉE, A.; DESAUTELS, C.; DOIZE, B. M.; FAIRBROTHER, J. $M$. Localization of the in vivo expression of $\mathrm{P}$ and $\mathrm{F} 1$ fimbriae in chickens experimentally inoculated with pathogenic Escherichia coli. Microbiology Pathogenesis, v. 22, p. 331-341, 1997.

34 PROVENCE, D. L.; CURTISS, R. Role of $\mathrm{crl}$ in avian pathogenic Escherichia coli: a knockout mutation of $\mathrm{crl}$ does not affect hemagglutination activity, fibronectin binding, or curli production. Infection and Immunity, v. 60, p. $4460-4467,1992$.

35 NGELEKA, M.; BRERETON, L.; BROWN, G.; FAIRBROTHER, J. M. Pathotypes of avian Escherichia coli as related to tsh-, pap-, pil-, and iuc-DNA sequences, and antibiotic sensitivity of isolates from internal tissues and the cloacae of broilers. Avian Diseases, v. 46, p. 143-152, 2002.

36 MENÃO, M. C.; FERREIRA, C. S. A.; CASTRO, A. G. M.; KNÖBL, T.; FERREIRA, A. J. P. Sorogrupos de Escherichia coli isolados de frangos de corte com doença respiratória crônica. Arquivos do Instituto Biológico, v. 65, n. 4, p. 15-17, 2002. 